\title{
Intérêt d'une double voie d'abord dans la chirurgie caverneuse complexe
}

\author{
M. SCHOUMAN
}

Centre d'Urologie, d'Andrologie et de Sexologie, 92200 Neuilly, France

\section{RESUME}

La fibrose des corps caverneux est responsable d'une impuissance organique lorsque les lésions sont étendues.

La restauration de l'activité sexuelle, et notamment des érections, passe par la mise en place d'une prothèse pénienne.

Cette chirurgie est rendue difficile car il existe alors des modifications anatomiques importantes, avec induration et rétraction, pas toujours régulière, des corps caverneux.

L'utilisation d'une double voie d'abord permet, au prix d'un risque infectieux potentiellement accru, une exposition totale, une implantation et une reconstruction plus aisées.

L'étude de cette série limitée (19 patients) permet de dégager les causes, la technique, les précautions opératoires et péri-opératoires, les résultats, dans la prise en charge chirurgicale de ces fibroses graves des corps érectiles.

Mots clés : Fibrose caverneuse, Maladie de La Peyronie, Priapisme, Prothèse pénienne, Traumatisme du corps caverneux.

\section{INTRODUCTION}

La chirurgie caverneuse complexe doit répondre a des impératifs techniques généraux et spécifiques : généraux, ce sont ceux liés à toute chirurgie caverneuse prothétique; spécifiques, ce sont ceux liés à la présence d'une fibrose rétractile, plus ou moins étendue et irrégulière, ce qui impose des choix spécifiques quant au type de prothèse et quant à la voie d'abord.

La double voie d'abord ici décrite permet le contrôle complet des deux corps caverneux, donc l'évaluation et le traitement à ciel ouvert des lésions.

Cela évite l'imagerie préopératoire dont on connaît les incertitudes, et le forage aveugle.

L'analyse de cette série de 18 cas confirme l'intérêt de cette approche originale dans des indications précises.

\section{METHODE}

L'antibiothérapie est commencée quatre heures avant l'intervention (délai nécessaire à une bonne imprégnation cutanée). Elle comporte une association d'aminoside et de bêta-lactamine par voie parentérale.

Le patient est rasé en salle d'opération, puis installé en position "gynécologique" et badigeonné à la BETADINE*.

Double paire de gants pour l'opérateur et l'assistant, la paire superficielle étant changée à chaque temps opératoire (après mise en place des champs, avant manipulation et mise en place de la prothèse). 
On réalise une première incision sub-coronale, $2 \mathrm{~mm}$ sous la couronne balanique. Le fourreau est progressivement décollé et récliné jusqu'à la base de la partie mobile du pénis. A ce niveau, on dissèque le ligament suspenseur qui est sectionné (comme dans la chirurgie d'allongement de la verge) [2]. Ce temps est important pour permettre ensuite l'extériorisation correcte de la verge.

On réalise ensuite une incision périnéale en "chapeau de gendarme" comme pour une urétroplastie bulbaire.

La verge est alors extraite de son fourreau et extériorisée par la plaie périnéale.

Cette manœuvre clé permet d'exposer la totalité des corps caverneux de la racine à l'extrémité.

Le repérage de l'urètre par une sonde est souvent confortable.

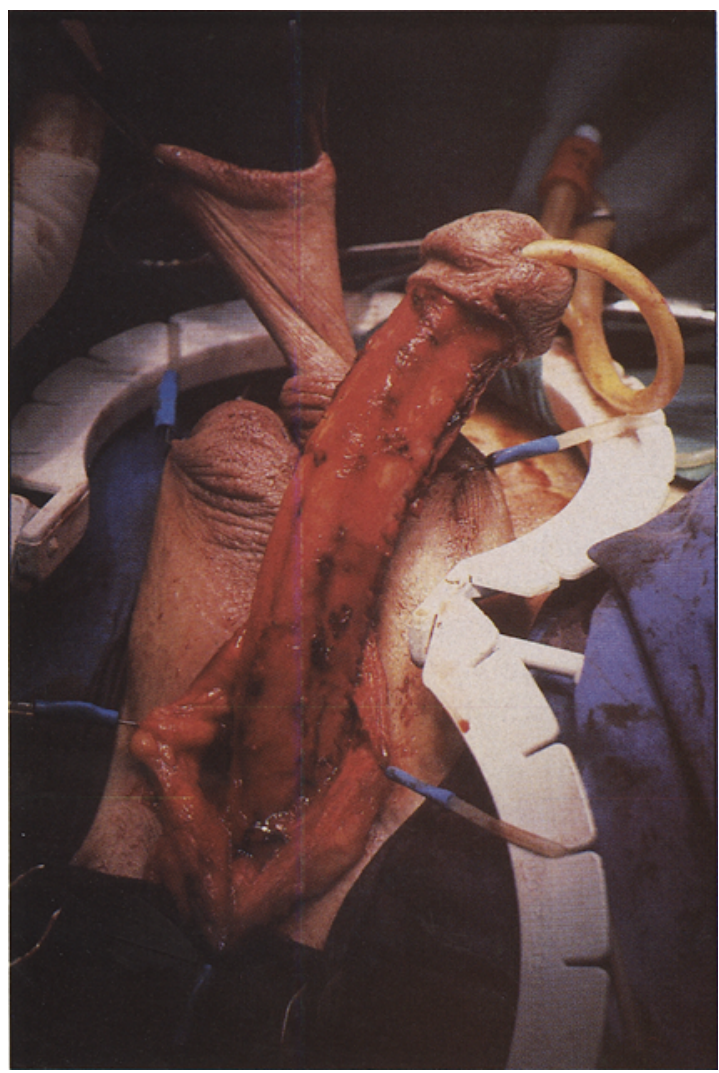

Figure $1:$ La verge extraite de son fourreau est totalement accessible par voie périnéale.
Les corps caverneux sont alors incisés sur toute la longueur nécessaire, les lésions de fibrose excisées à la demande.

La prothèse est ensuite choisie en fonction de la longueur et de la largeur disponibles. On utilise le plus souvent des prothèses semirigides ou malléables, de petit diamètre.

La prothèse est couchée dans le lit caverneux et fixée en bonne place par des fils tressés non- résorbables. La zone opératoire est alors imprégnée de RIFOCINE*.

La prothèse doit ensuite être couverte. Cependant le défect albuginéal ne permet habituellement qu'une couverture partielle. On complète donc par un lambeau de fascia de Buck ou de prépuce, si le défect est limité. Dans le cas contraire on préfere utiliser un greffon semi synthétique résorbable de type URO-PATCH, ou une plaque de GORETEX.

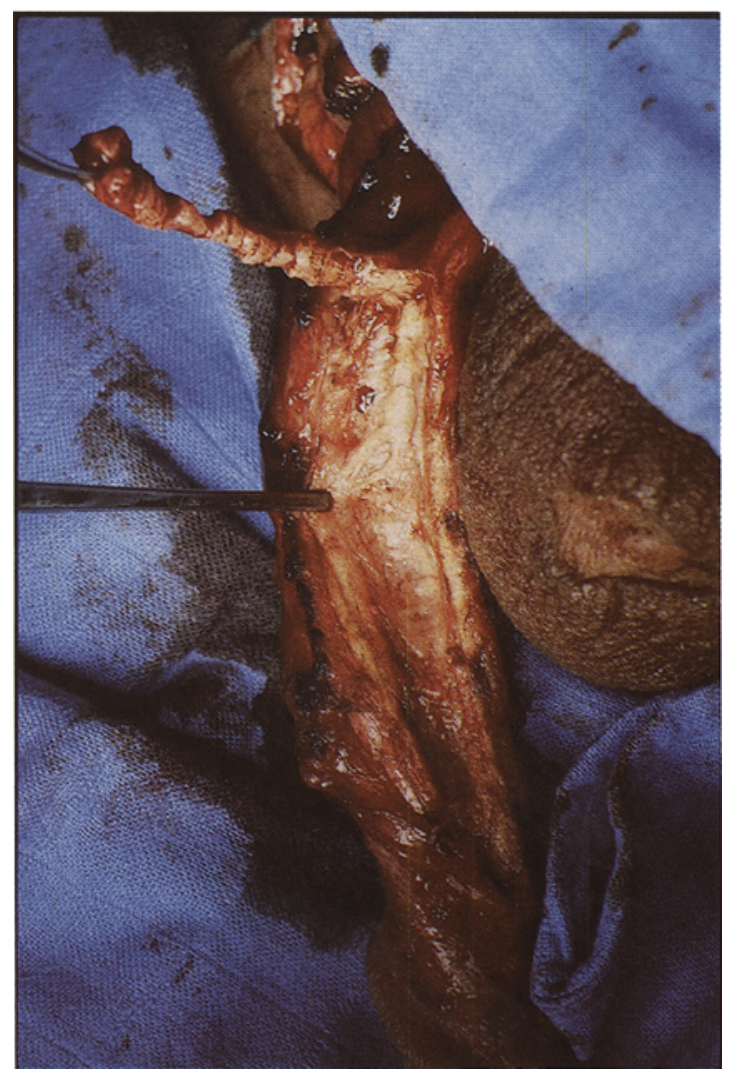

Figure 2 : Excision de la fibrose caverneuse. 


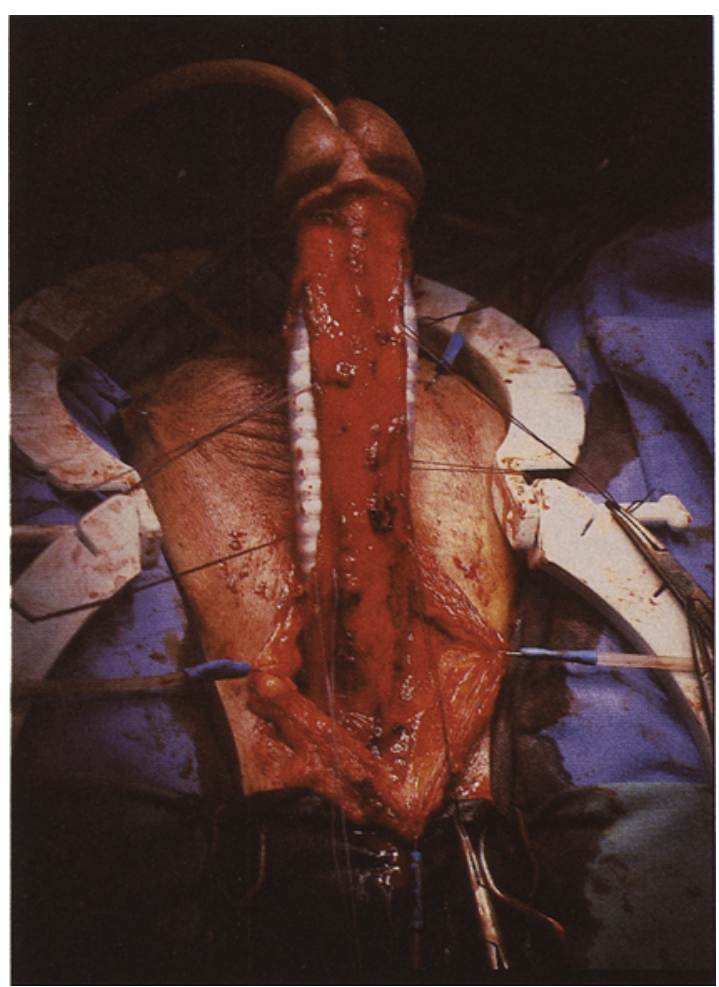

Figure 3 : Mise en place des prothèses péniennes.

La verge est ensuite réintroduite dans le fourreau cutané, le prépuce réséqué à la demande pour permettre une suture sans traction au niveau balanique.

La conservation du prépuce expose au risque de phimosis, de para-phimosis, d'œdème préputial post-opératoire prolongé, pouvant nécessiter un geste complémentaire.

La plaie périnéale est fermée plan par plan au fil résorbable sur un drainage aspiratif.

Si l'on craint des troubles mictionnels post opératoire, on préférera la mise en place d'un cathéter sus-pubien au maintien de la sonde urétro-vésicale.

Outre les soins locaux habituels dans la période post opératoire, la double antibiothérapie est poursuivie pendant cinq jours, puis une bêta-lactamine seule pendant 3 semaines.

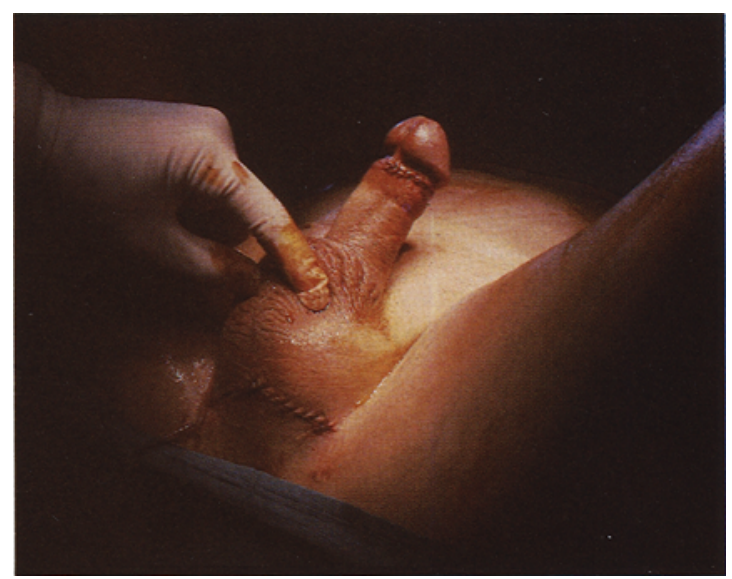

Figure 4 : Aspect en fin d'intervention.

La reprise de l'activité sexuelle n'est pas autorisée avant un mois, après consultation de contrôle.

\section{MATERIEL}

19 patients ( 32 à 67 ans)ont été opérés de Juin 90 à Décembre 96.

Les étiologies étaient représentées par :

- rejet de prothèse

11 cas

- traumatisme

1 cas

- fibrose iatrogène (post IIC)

4 cas

- maladie de La Peyronie

3 cas

Parmi les rejets de prothèse, on compte 9 gonflables, 1 malléable, 1 semi-rigide. Lors de la consultation, tous les patients étaient explantés depuis plus de 3 mois:

- 1 explantation à $\mathrm{J} 1$ pour problème technique (?)

- 1 rejet à $\mathrm{J} 13$ par une ulcération urétrale distale.

- 1 explantation pour douleurs intolérables.

- 1 double rejet après tentatives par deux équipes différentes.

- 6 rejets par infection post-opératoire précoce. Le protocole lavage-irrigationréimplantation précoce, qui permet, en principe, d'éviter la rétraction cicatricielle n'avait été tenté chez aucun des patients qui nous ont été adressés. 
- 1 explantation tardive ( 2 ans) pour insatisfaction (prothèse gonflable).

En ce qui concerne le cas de fibrose caverneuse post-traumatique, il ne s'agissait pas d'une rupture caverneuse simple (type faux-pas du coït), mais d'une lésion survenue dans un contexte de traumatisme du bassin. La réparation caverneuse a pris place après la réparation des lésions ostéoarticulaires et urétrales.

Les fibroses iatrogènes post- IIC ont concerné 2 fois des injections de Papavérine* (1 fois chez un patient qui pratiquait ce type d'injections à des doses de 10 et $15 \mathrm{mg}, 1$ fois chez un patient qui avait subi une pharmaco-cavernosométrie après IIC de 40 mg de Papavérine*). Les deux autres patients, ont présenté un priapisme après IIC de Moxisilite $(20 \mathrm{mg})$ et de PGE1 (10 $\mu \mathrm{g})$. Dans les deux cas il s'agissait d'une première injection "test".

Les 3 cas de maladie de La Peyronie présentaient des lésions étendues, intéressant, en outre, la racine des corps caverneux. L'écho-doppler et l'IRM, chez ces 3 patients, n'ont fait que confirmer les données cliniques.

\section{RESULTATS}

L'évolution post opératoire a été simple chez 18 de nos 19 patients.

Le contrôle clinique a eu lieu à 1 et 3 mois.

La reprise d'activité sexuelle, bien qu'autorisée dès la fin du premier mois, n'a eu lieu qu'autour du troisième mois.

13 patients ont été revus à 1 an avec un bon résultat cosmétique (au prix d'un raccourcissement important pour l'un d'entre eux), et fonctionnel.

1 patient n'a pas été revu.

4 patients ont un recul inférieur à 1 an.

1 patient a présenté un rejet (mécanique) au deuxième jour par masturbation "précoce intempestive". Il s'agissait d'un patient qui avait présenté un premier rejet de prothèse 7 mois au préalable dans un contexte d'infection à pyocyanique.

\section{DISCUSSION}

L'utilisation d'une double voie d'abord, subcoronale et périnéale, qui permet un abord de la totalité des deux corps caverneux, est intéressante dans la chirurgie prothétique pénienne complexe.

Elle permet un bon bilan des lésions et une reconstruction sous contrôle visuel. Ceci nous a semblé d'autant plus intéressant dans les lésions post-traumatiques que cet abord permet un contrôle simultané de l'urètre.

L'incision périnéale peut faire redouter un risque septique.

Les précautions draconiennes d'asepsie, le protocole d'antibiothérapie, l'utilisation privilégiée de matériel résorbable contribuent à limiter ce risque.

Pour l'instant, nous n'avons à déplorer qu'un seul rejet. Cela tient sans doute au caractère encore limité et relativement récent de notre série. Encore ce rejet est-il intervenu chez un patient opéré après un premier rejet infectieux de prothèse (pyocyanique) et qui s'est masturbé le soir même de la deuxième intervention !

La rétraction de la verge est quasi constante dans ce type de lésions. Nous n'avons pas cherché à la corriger. Le ligament suspenseur, systématiquement sectionné dans cette voie d'abord, n'a jamais été réinséré. Son absence n'a eu aucune conséquence fonctionnelle néfaste, mais sa section n'a pas non plus permis un allongement de la verge tel qu'on le réalise dans la chirurgie d'agrandissement. Cela tient au fait que nous n'avons pas souhaité associer la plastie cutanée qui aurait alors nécessité une troisième incision. Cette constatation est 
d'ailleurs conforme aux observations rapportées par Bondil chez le cadavre [2]. La troisième incision, qui se serait trouvée en zone pré- et sous-pubienne aurait alors présenté un risque septique et vasculaire que nous n'avons pas voulu courir.

En revanche, dans tous les cas, même lorsque le corps caverneux se résumait à un cordon fibreux, l'insertion prothétique a toujours été possible avec cette méthode.

Le seul patient que nous avons (provisoirement ?) repoussé, est un patient jeune, présentant des séquelles d'écrasement du bassin, porteur d'une colostomie (risque septique) et d'une arthrodèse d'une hanche avec des rétractions tissulaires du pli génito-crural (impossibilité d'installation en position gynécologique). Si la décision est prise, un seul corps de prothèse pourra être implanté, ce qui ne constitue pas, en soi, une contre-indication.

\section{CONCLUSION}

Lorsqu'on est confronté à une fibrose caverneuse étendue, on n'a d'autre ressource thérapeutique que la mise en place d'un implant pénien. Cependant, cette chirurgie peut être rendue difficile par l'étendue des lésions fibreuses et la rétraction plus ou moins régulière dans toutes les dimensions. Ce type de lésions s'observe après priapisme, notamment compliquant une injection intra-caverneuse (IIC), dans certaines maladies de La Peyronie très évoluées, dans un contexte post-traumatique tardif, et dans les suites tardives du rejet d'une première implantation prothétique.

Les lésions de fibrose caverneuse sont difficiles à documenter de façon précise et le parallélisme radio-clinique incertain, ce qui peut rendre difficile le choix de la voie d'abord [1].

Le forage aveugle par les dilatateurs de Rossello n'est pas dénué de risques, en particulier de fausse route.
C'est pourquoi nous avons choisi d'appliquer à la reconstruction prothétique des corps caverneux la double voie d'abord décrite pour la chirurgie urétrale [3].

Cette voie d'abord donne un excellent jour sur l'ensemble des corps caverneux. Cela permet dans tous les cas de fibrose étendue des corps caverneux, quelle qu'en soit la cause, l'implantation d'une prothèse malléable ou semi-rigide avec un bon résultat fonctionnel.

Les écueils, relatifs, sont le risque septique et la non-correction de la rétraction de la verge avec persistance du raccourcissement préopératoire.

Néanmoins, la restauration de la sexualité, chez des patients qui avaient perdu tout espoir, nous semble justifier le risque d'échec ou d'imperfections que représente cette intervention.

\section{REFERENCES}

1. AUSTONI E. : Use of dynamic NMR in diagnostic evaluation of penile pathologies : Int.J.Impotence Res. : 1990, 2, supplement 2, 161.

2. BONDIL P., DELMAS V. : Allongement de la verge par section du ligament: Etude sur le cadavre : $1^{\circ}$ congrès de l'European Society for Impotence Research (ESIR), 1995.

3. DE SY W.A., OOSTERLINCK W. : Atlas de chirurgie reconstructive urétrale. R.Franceschinis Ed. : 1990, 28.

4. SCHOUMAN M. : Utilisation d'une double voie d'abord, sub coronale et périnéale, dans la chirurgie pénienne complexe: 1994, Deauville, Congrès de la Société d'Andrologie de Langue Française (SALF), (prix du meilleur poster).

\section{ABSTRACT \\ Double approach for difficult cavernous surgery}

M. Schouman

Extensive cavernous body fibrosis is responsible for organic impotence when the lesions have advanced. 
Those fibrotic lesions can be caused by penile trauma, severe peyronie's disease, priapism (mostly post ICI) and rejection of a previously implanted prosthese.

Sexual activity can be restored, mainly the erections, and is posiible with the insertion of penile prosthese. As a result of important anatomical changes, due to irregular indurations and retractions of the corpora, this surgery can be very difficult.

The use of a double incisional approach (sub-coronal and perineal, degloving the penis out of its shaft through the perineal incision), allows, inspite of a potentially increa- sed risk of infection, a total exposure making implantation and reconstruction much easier.

The study of this limited series (from 1990 to 1996,19 patients were operated with this procedure), allows to draw some preliminary conlusions about the pathology, the technique, the operatory (pre-, per-, and post-) precautions of this surgical procedure that treats serious erectile tissue fibrosis. Results are satisfactorily concerning recovery of penile rigidity as well as sexual activity.

Key words : Cavernous fibrosis, Cavernous trauma, Penile prosthese, Peyronie's disease, Priapism. 